\title{
Optimization and sensitivity enhancement of high-resolution molecular resist for EUV lithography
}

\author{
Andreas Frommhold*a ${ }^{* a}$ Alexandra McClelland ${ }^{\mathrm{b}}$, John Roth $^{\mathrm{c}}$, Roberto A. Fallica $^{\mathrm{d}}$, Yasin Ekinci ${ }^{\mathrm{d}}$, \\ Alex P. G. Robinson ${ }^{\mathrm{a}}$ \\ ${ }^{\text {a }}$ School of Chemical Engineering, University of Birmingham, B15 2TT Birmingham, UK \\ ${ }^{\mathrm{b}}$ Irresistible Materials Ltd., Unit 4, Chelhydra Walk, Marina, SA1 YAG Swansea, UK \\ ${ }^{\mathrm{c}}$ Nano-C Inc., 33 Southwest Park, Westwood, MA 02090, USA \\ ${ }^{\mathrm{d}}$ Laboratory for Micro and Nanotechnology, Paul Scherrer Institute, 5232 Villigen, Switzerland
}

\begin{abstract}
We have recently introduced a new molecular resist system that demonstrates high-resolution capability. A series of studies such as quencher choice and loading was conducted in order to optimize the performance of this material. The optimized conditions allowed patterning $14 \mathrm{~nm}$ half-pitch (hp) lines with a line width roughness (LWR) of $3.56 \mathrm{~nm}$ at the XIL beamline of the Swiss Light source. Furthermore it was possible to resolve $11 \mathrm{~nm}$ hp features with $5.9 \mathrm{~nm}$ LWR. First exposure results on an NXE3300 are also presented. We have also begun to investigate the addition of metals to EUV photoresist as a means to increase sensitivity and modify secondary electron blur. Initial results for one of the metal additives show that the sensitivity could be enhanced by up to 60 percent.
\end{abstract}

Keywords: EUV Lithography, Molecular Resist, Resist Sensitivity

\section{INTRODUCTION}

The International Technology Roadmap of Semiconductors (ITRS) lists EUV lithography (EUVL) as one of the most promising candidates in replacing current integrated circuit manufacturing processes for the transition to future technology nodes. However unresolved still remain - such as low available source power. Using a much shorter wavelength for patterning has also made the development of new photoresist platforms necessary, a challenging task in itself.

Significant research into new materials has been undertaken, but to date no photoresist has been able to simultaneously meet all the specifications laid out in the ITRS. In addition to fulfilling the current resist targets for the next generation of devices; new material platforms must also have the potential to meet the outlined specifications beyond that point, to ensure a useful lifespan for next generation lithography. Recent interest in novel metal-based resist materials [1-3] has increased as a possible route to improved sensitivity at high resolution. But concerns about integration of all-metal resists still persist.

Our focus is on developing a molecular resist platform for EUVL application. Recently we had synthesized a new resist molecule that showed good photo speed, low line edge roughness (LER) and high resolution patterning. Here we are reporting our ongoing efforts to optimize the formulation and process conditions to further improve the performance of this material. As the patterning dose of resists typically increase as the feature size decreases we have also begun to explore the addition of metal containing compounds to our resist that ideally do not impact the pattern quality while improving the sensitivity and first results are presented here.

\section{EXPERIMENTAL}

The resist samples were prepared by dissolving the individual components in ethyl lactate. They were then combined in various weight ratios and concentrations. The resist was spun onto a proprietary carbon underlayer that we have developed consisting of a mixture in equal parts of a fullerene derivative and an epoxy crosslinker. Prior to the application of the underlayer the silicon substrates were cleaned in a three-step process: $10 \mathrm{~min}$ immersion in isopropyl

Extreme Ultraviolet (EUV) Lithography VII, edited by Eric M. Panning, Kenneth A. Goldberg. Proc. of SPIE Vol. 9776, 977614 - @ 2016 SPIE · CCC code: 0277-786X/16/\$18 · doi: 10.1117/12.2219221 
alcohol (IPA) in an ultrasonic bath, 10 min immersion in piranha solution $\left(1: 1 \mathrm{H}_{2} \mathrm{SO}_{4}: \mathrm{H}_{2} \mathrm{O}_{2}\right)$ and finally 2 min dip in a weak aqueous solution of hydrofluoric acid $(0.1-1 \%)$.

After spin coating of the resist the samples received a post application bake. All EUV exposures were performed using the XIL-II interference lithography tool at the Paul Scherrer Institute, Switzerland [4], except for the results in section 3.3, which were performed with a NXE3300 scanner at the Interuniversity Micro Electronics Centre (IMEC) in Leuven, Belgium. After exposure the samples received a post exposure bake and were developed in n-butyl acetate for 60 seconds followed by an IPA rinse.

Exposed samples were analyzed with a scanning electron microscope (SEM) in top-down view. Critical dimension (CD) and LER were calculated from the SEM images with the commercial software package SUMMIT. As the XIL tool does not allow direct measurement of the dose at the wafer, the exposure was measured with respect to an internal PSI reference resist calibrated against exposures of the same resist at the Intel MET [5]. For features smaller than the capability of the calibration resist, dose was estimated from higher pitch sizes.

\section{RESULTS}

The baseline material for the optimization efforts is the previously introduced xMT resist [6]. It is a blended formulation of xMT resin, molecular epoxy crosslinker and a sulfonium photoacid generator mixed in 0.2:2:1 weight ratio with the addition of $5 \%$ quencher in the form of a photo-decomposable nucleophile that controls epoxy crosslinking [7]. The chemical structures of the components are shown in figure 1.

\section{Molecular resin}

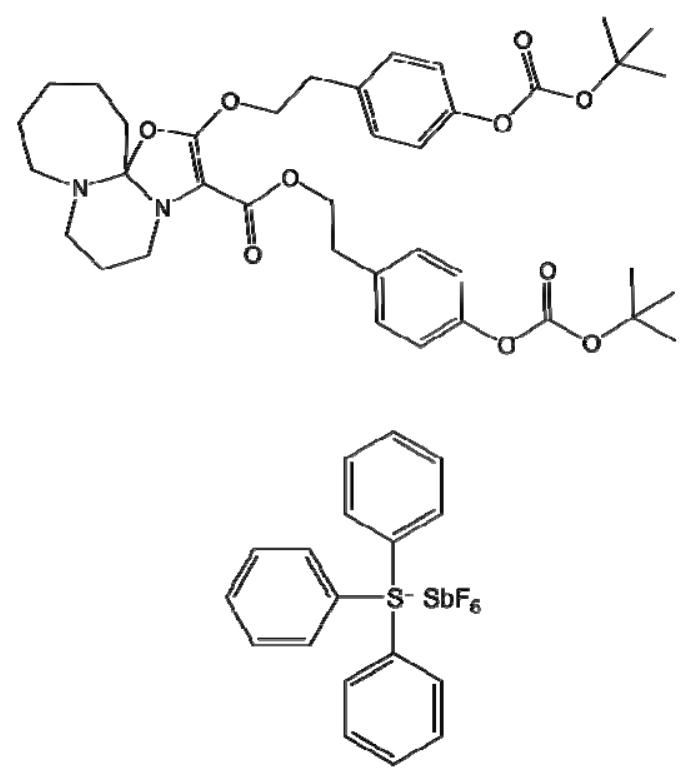

Photoacid generator
Crosslinker
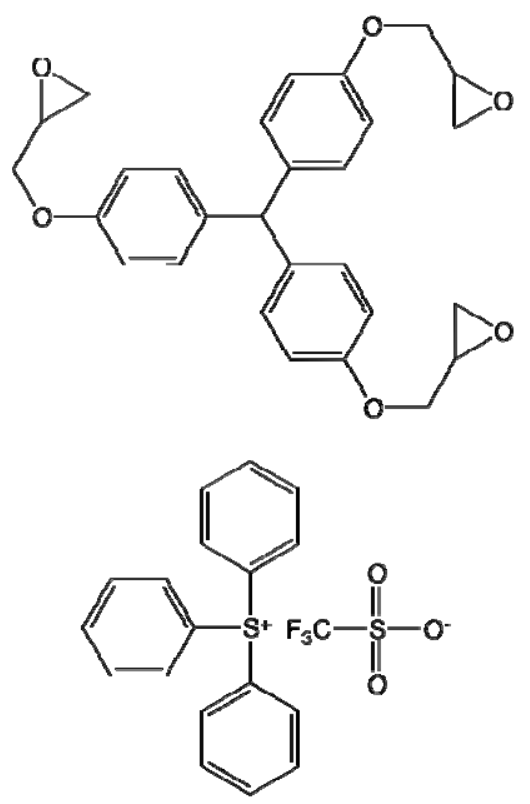

Quencher

Figure 1 Baseline resist components of $x M T$ resist

\subsection{Resist Optimization}

The first aspect of resist formulation examined was the choice of quencher and quencher loading. Four different quenchers were chosen: triphenyl sulfonium (TPS) triflate (PAG03-01), TPS nonaflate (PAG04-05), diphenyl iodonium tosylate (PAG11-07) and TPS tosylate (PAG11-09). The loading level was 5\% of the total weight of the resist in each case. Line-space patterns of $14 \mathrm{~nm}$ hp were printed and the dose-to-size as well as line edge roughness for each quencher compared. The results in figure 2 show that the compound TPS tosylate was most effective at quenching the xMT resist, 
as seen by the increased dose-to-size. This quencher also gave the best LER performance. Hence this compound was determined to be the optimum quencher choice for formulation of our resist.
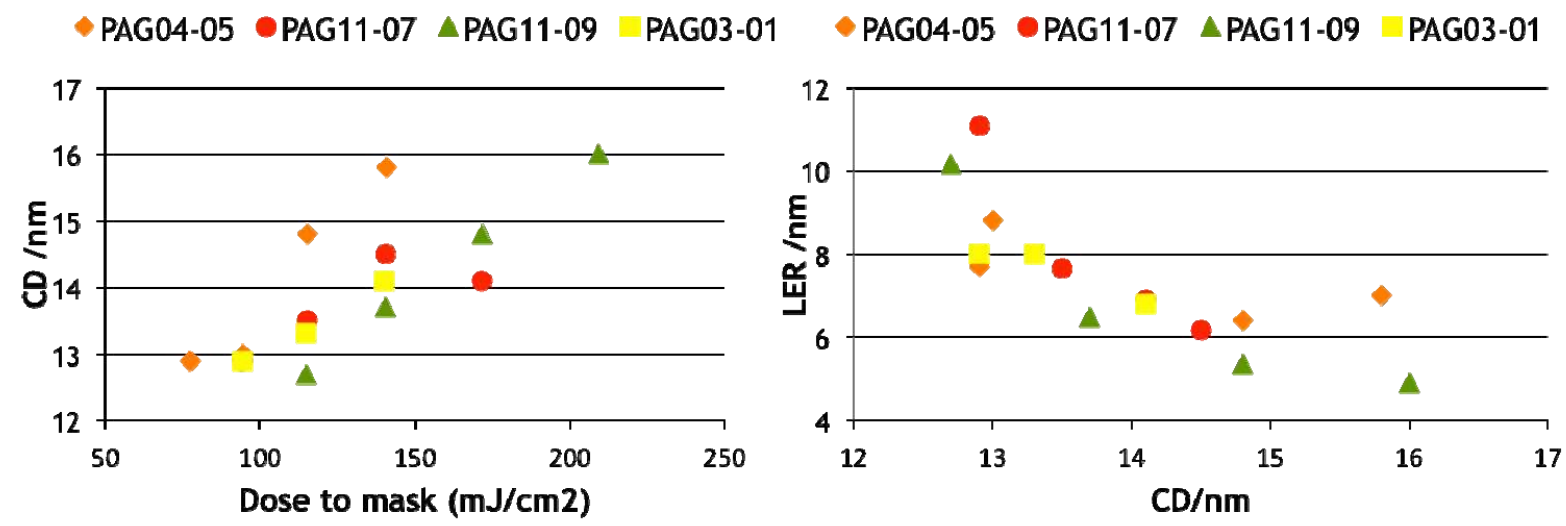

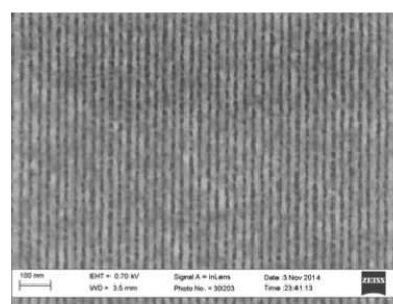

PAG04-05

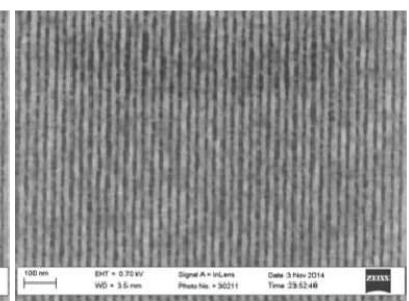

PAG11-07

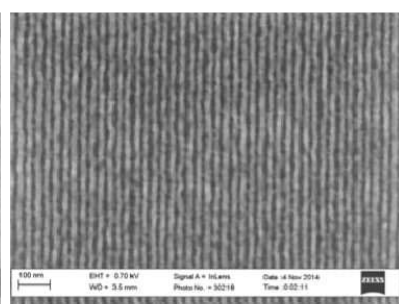

PAG11-09

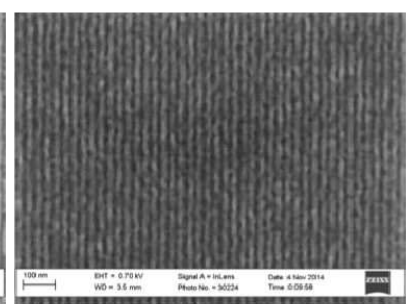

PAG03-01

Figure 2 Impact of quencher choice on resist performance
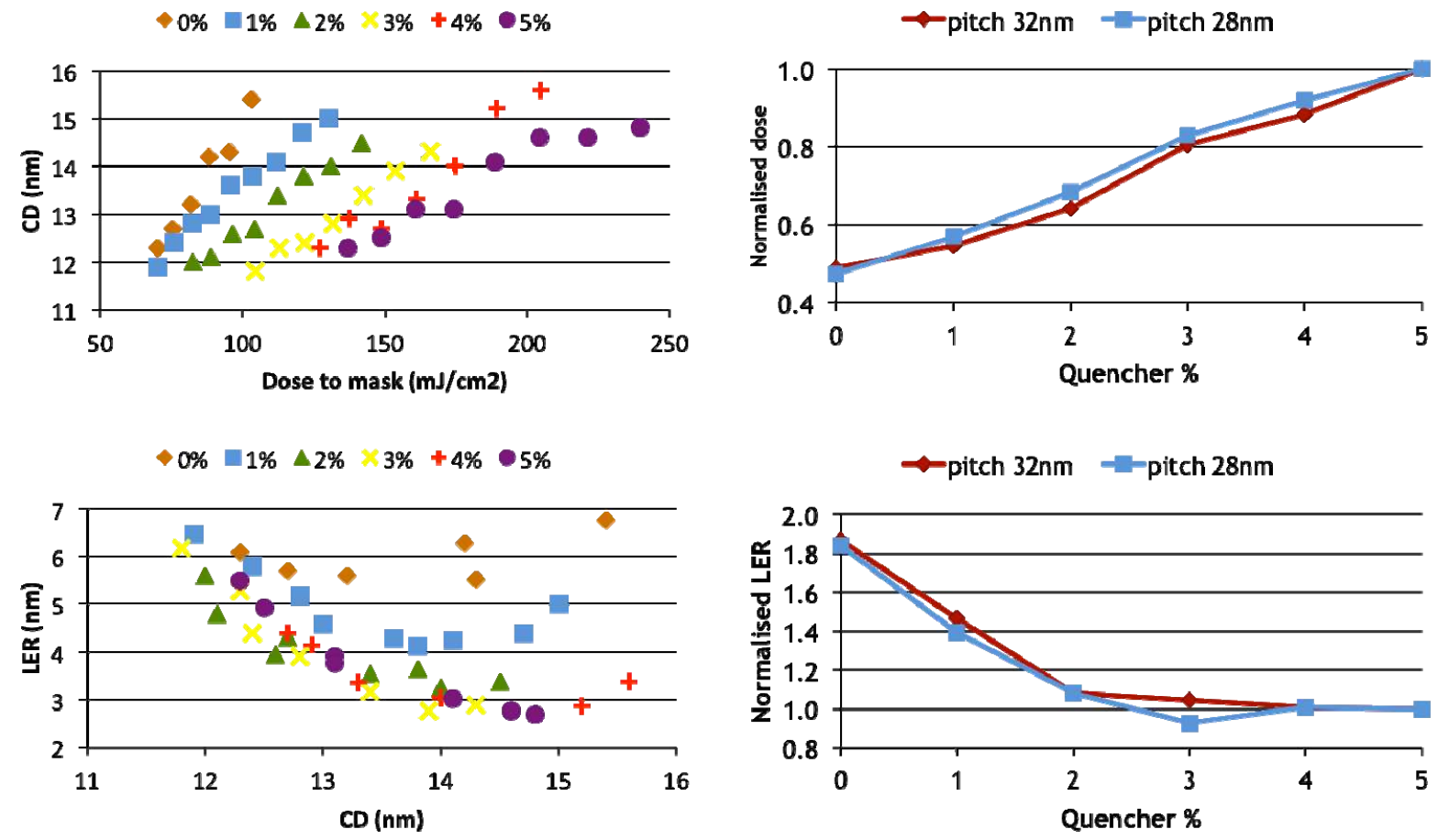

Figure 3 Effect of quencher loading level on resist performance 
Next the impact of quencher loading level was studied. In our previous work on fullerene containing resists with similar composition [8] we found a value of five percent to be the optimum level, and have to date used the same amount of quencher in the baseline xMT formulation. In order to establish the correct quencher level for the xMT resist, resist formulations with PAG11-09 quencher and the loading levels ranging from 0 to 5 percent were prepared and exposed. Figure 3 shows the corresponding plots of critical dimension (CD) and LER for each level. As expected the dose-to-size increases as more quencher is added to the formulation. Interestingly the LER however shows little sign of improvement beyond about 2 percent loading and levels above that generally follow the same curve. This cut-off behavior is clearly visible in the plot of the normalized LER vs quencher level with the slope of the graph drastically changing at the 2 percent point. Normalization is done with the value at 5 percent. It is therefore possible to improve resist sensitivity by choosing a lower quencher loading without decreasing the pattern quality, illustrated by the normalized dose vs quencher level graph and figure 4.

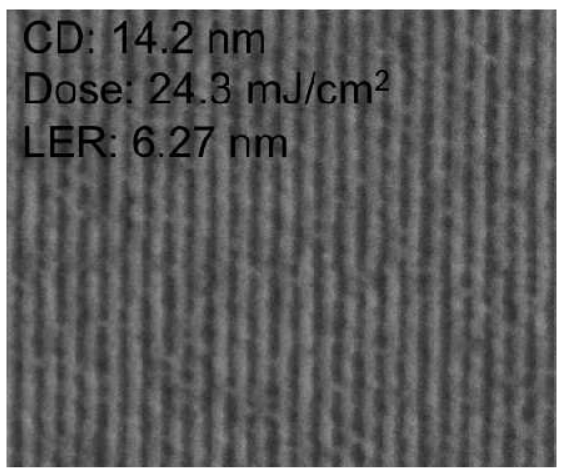

$0 \%$ quencher

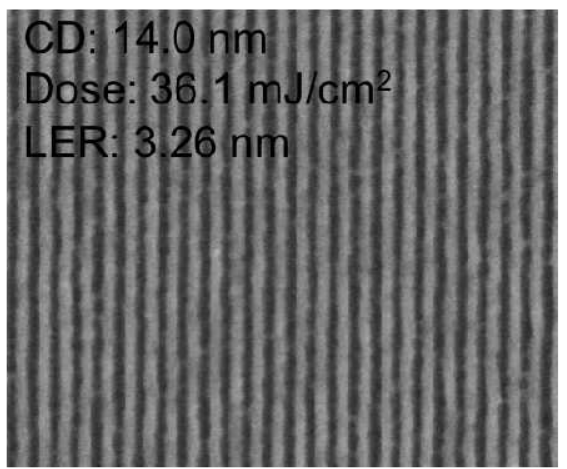

$2 \%$ quencher

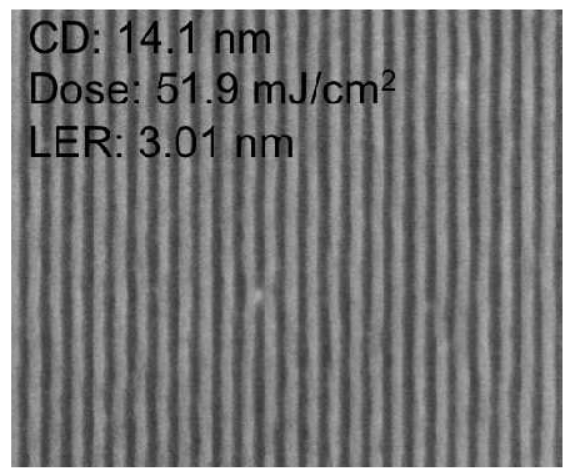

$5 \%$ quencher

Figure 4 Line-space patterns with $14 \mathrm{~nm}$ hp at three different quencher levels

Another issue that required optimization was the occurrence of speckle patterns on the substrate surface after development for larger substrate sizes. The rinse solvent used to wash off the developer was suspected to be the root cause for this residue. So far we have used IPA as our rinse material, but it has a high vapor pressure (40 $\mathrm{mm} \mathrm{Hg}$ ) making it volatile. During blow drying the IPA can evaporate leaving behind leftover resist material from the development process. Replacing the IPA with methyl isobutyl carbinol (MIBC), which has much lower vapor pressure (3 $\mathrm{mm} \mathrm{Hg}$ ), eliminated the residue as is shown in figure 5.

IPA Rinse
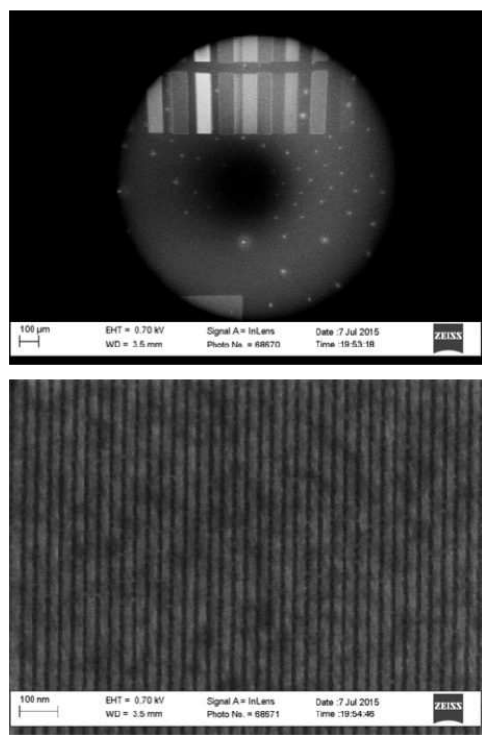

MIBC Rinse
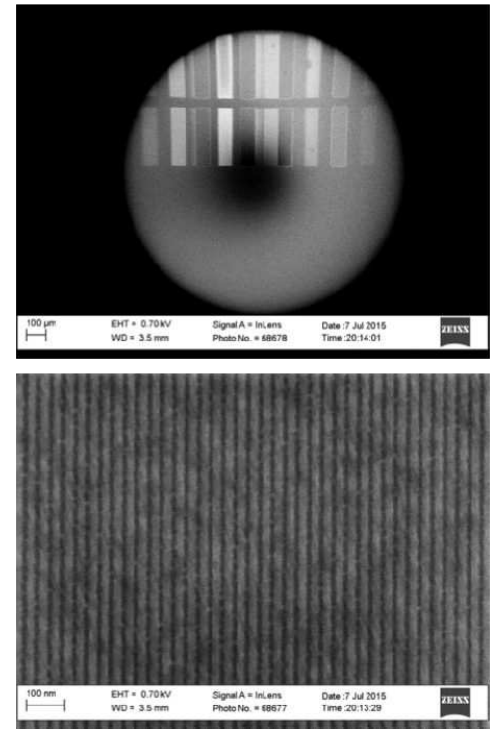

Figure 5 Comparison of IPA and MIBC as rinse solvent 
Applying the combined optimization strategies to the xMT resist greatly improved the pattern performance (figure 6). The LWR of the features reduced by more than $50 \%$ while maintaining the resolution capability at $14 \mathrm{~nm} \mathrm{hp}$. In addition features at $11 \mathrm{~nm} \mathrm{hp}$ are now clearly resolved, which previously only showed modulation. Features below $14 \mathrm{~nm} \mathrm{hp}$ suffer pattern collapse with the current formulation. Reducing the concentration and hence film thickness suppresses this issue (figure 7). Since there is a critical limit to film thickness for subsequent pattern transfer, it is not sustainable for even smaller feature sizes. Therefore we are currently developing a new underlayer that actively binds to the resist in order to avoid pattern collapse and enable further down scaling.

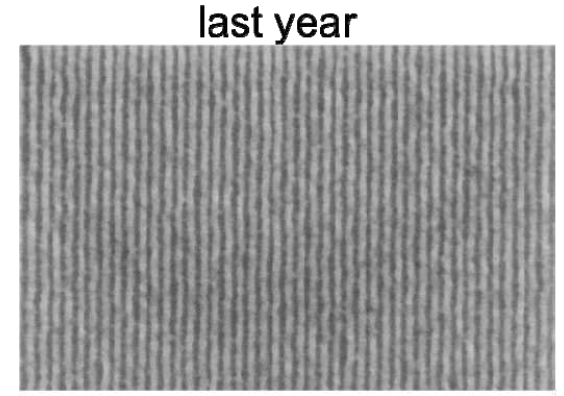

$13.9 \mathrm{~nm}, 8.07 \mathrm{~nm}$ LWR

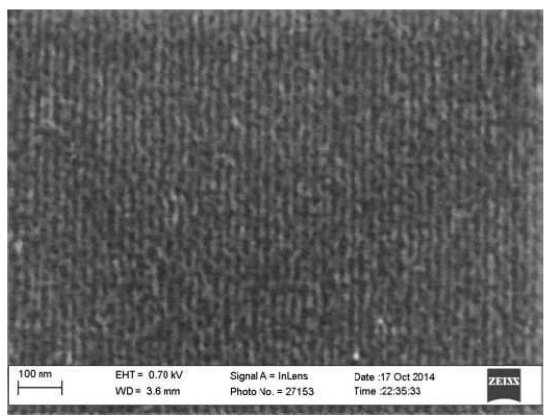

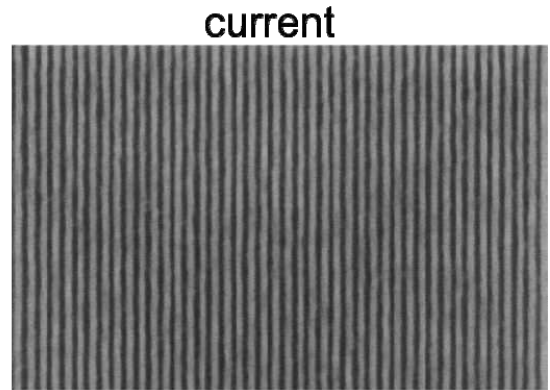

$13.9 \mathrm{~nm}, 3.56 \mathrm{~nm}$ LWR

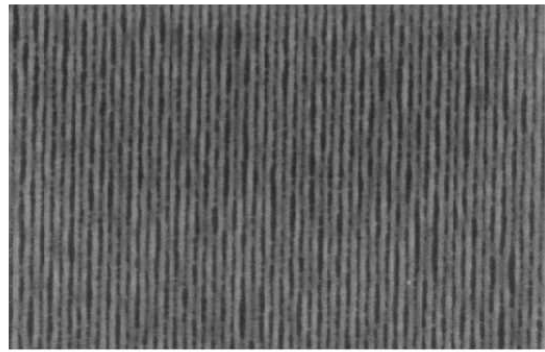

$11.9 \mathrm{~nm}, 5.9 \mathrm{~nm}$ LWR

Figure 6 Comparison of last year's xMT with current optimized version
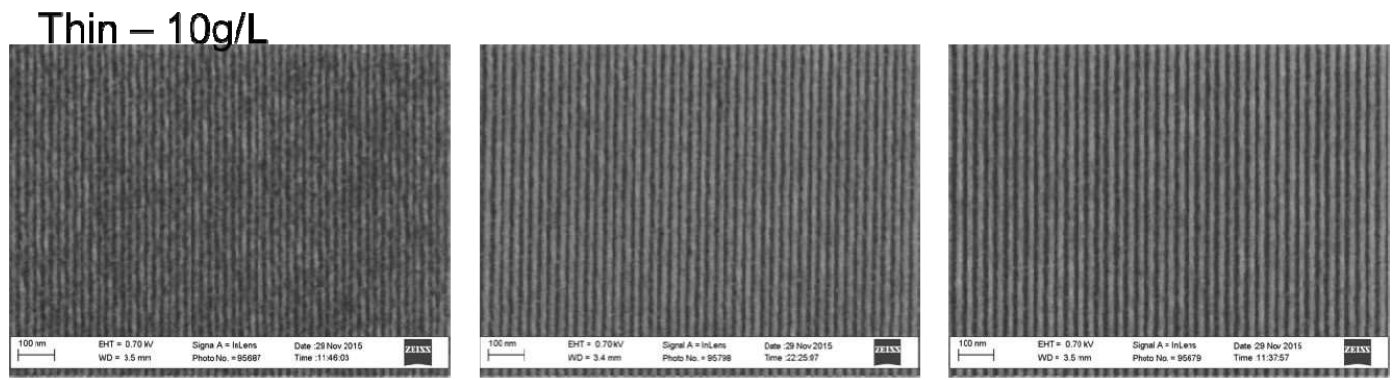

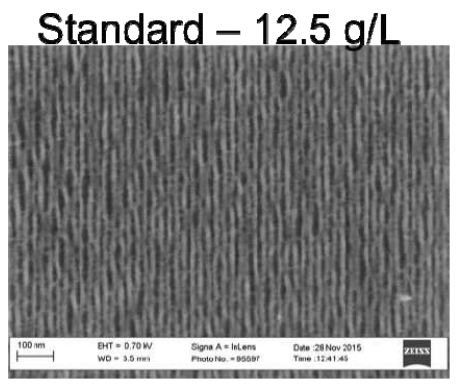

$11 \mathrm{~nm} \mathrm{hp}$

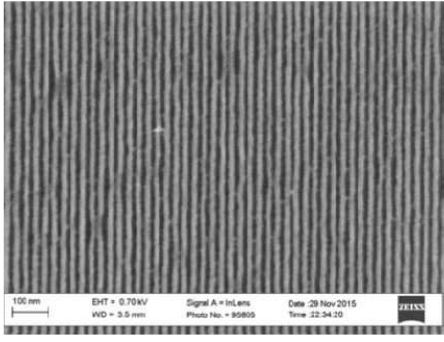

$13 \mathrm{~nm} \mathrm{hp}$

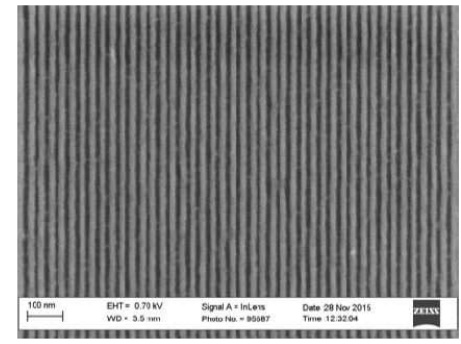

$14 \mathrm{~nm} \mathrm{hp}$

Figure 7 Elimination of line 'wiggle' 


\subsection{Effect of xMT}

Last year we reported on experiments to gain better understand of the role xMT plays in the resist. We have continued our investigation and the latest results are shown in figure 8. Since xMT is an amalgamation of a 1,8-diazabicycloundec7-ene (DBU) molecule linked to a tert-butyl carbonyl protected phenol via a malonate chain, resist batches were prepared with either DBU or a mixture of DBU and xMT precursor taking the place of xMT in the formulation. While the DBU only version was the least sensitive, xMT showed the best LER. The DBU-precursor mixture was the most sensitive but showed the worst LER performance. Of particular interest is the direct comparison of xMT and DBU:precursor as both are identical in their make-up the only difference being that in xMT the DBU and the precursor are linked together. Nonetheless the xMT produces significantly better printed lines. The binding of the DBU appears to be a crucial factor. The functional groups on the xMT facilitate the incorporation into the polymerized epoxy network. It is than possible that the xMT acts as an analogue to a polymer bound photoacid generator (PAG), but instead of a PAG it is a quencher that is bound to the polymer.
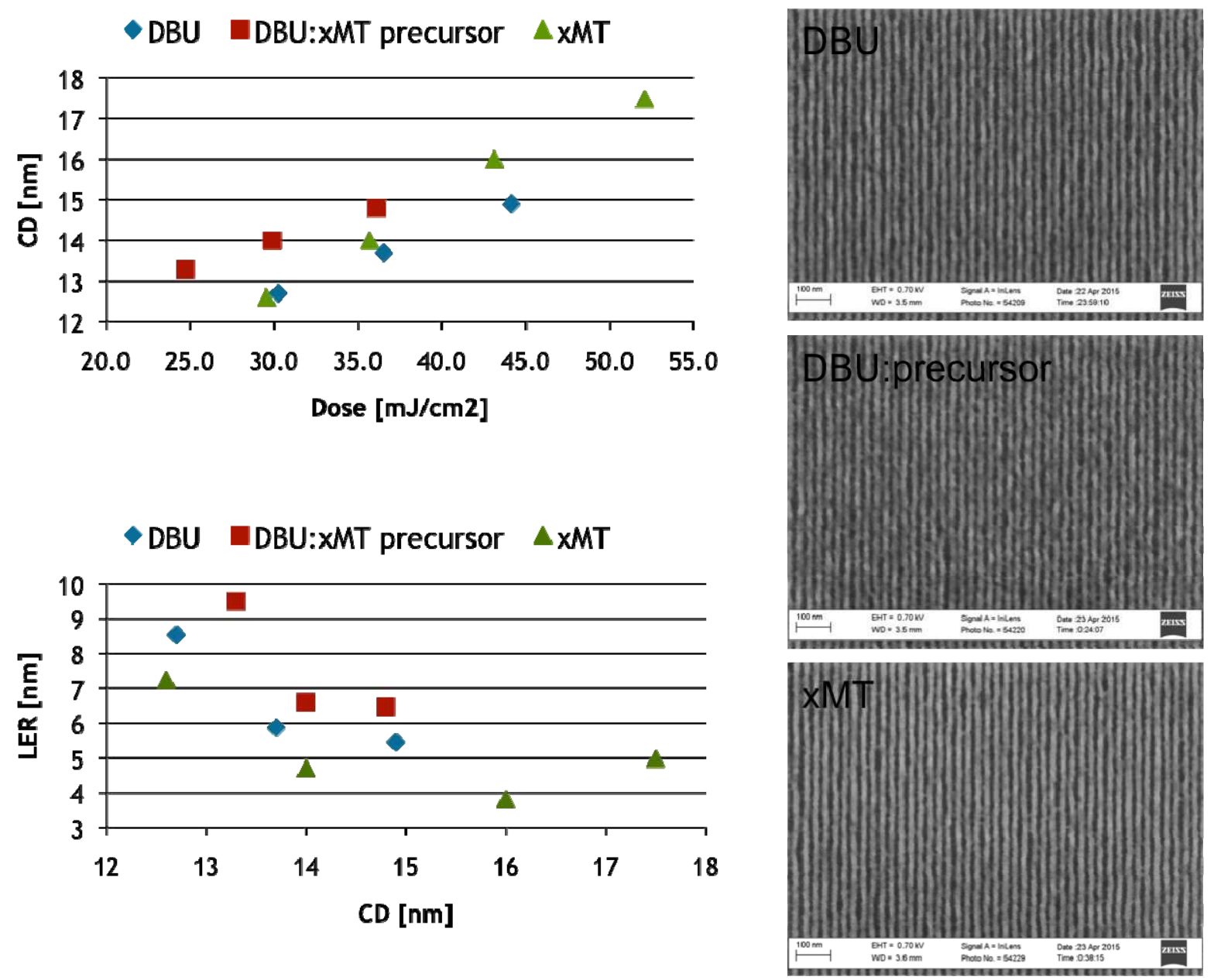

Figure 8 Effect of xMT on patterning performance

\subsection{NXE3300 exposure}

A version of the xMT resist has been exposed for the first time on a NXE330 at IMEC (figure 9). Features down to 19 $\mathrm{nm} \mathrm{hp}$ were well resolved. Below that bridging and collapse dominated the patterns. The exposure dose of $34 \mathrm{~mJ} / \mathrm{cm}^{2}$ corresponds well with the doses seen in the tests at PSI. The LWR of the patterns was around $4 \mathrm{~nm}$. The resist was exposed on bare silicon without the proprietary carbon underlayer. Comparison at PSI showed that the use of the carbon underlayer reduces the LWR of the material. From previous experience the film thickness chosen to be $32 \mathrm{~nm}$ here is 
known to be prohibitive for patterning in the sub-18 nm regime for this material. For possible future exposures a thinner film and optimized resist will be used to improve the pattern resolution.

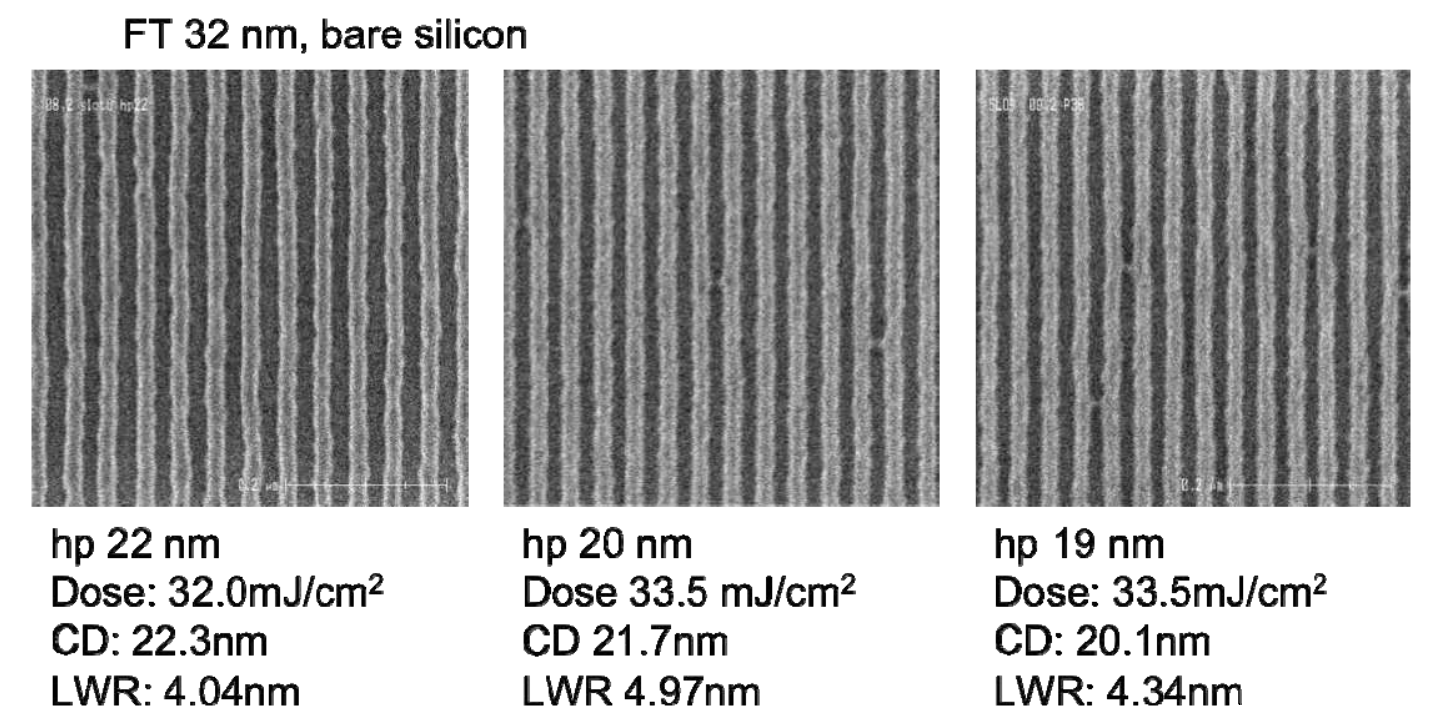

Figure 9 First exposure results from NXE3300

\subsection{Metal addition}

Metal-based resists are currently receiving much attention. Metals do often have a higher absorption at $13.5 \mathrm{~nm}$ wavelength radiation compared to organic resist materials. Higher absorption is a potential route to overcome the photon shot noise limit in EUV lithography as well as significantly improve sensitivity to enable high volume manufacturing at current source power output. While many efforts focus on the higher absorption properties of metals, there is another aspect to metals as well. Fundamental studies are currently under way about the different nature of light-resist interaction in the EUV range, but electron scattering properties are thought to be an important part of this. For example they account for the secondary electron blur. If one compares the elastic and inelastic interaction of carbon, the most abundant species in organic resists, with for instance platinum (figure 10), it is evident that the interactions in the two differ substantially in the range of interest for EUV lithography $(10-92 \mathrm{eV})$. This analysis can be done for most metals and classified into a variety of behaviors. We intend to study this in more detail in the resist. However, it is not practical to attempt to synthesis a new resist for each metal species. Therefore we devised the concept of metal additives, chemical compounds that contain the metal species of interest that are blended into the xMT resist. In a first instance this concept is shown as a sensitizer to increase the sensitivity of the xMT but in further work we intend to screen for the differences in resist electron interaction as well.
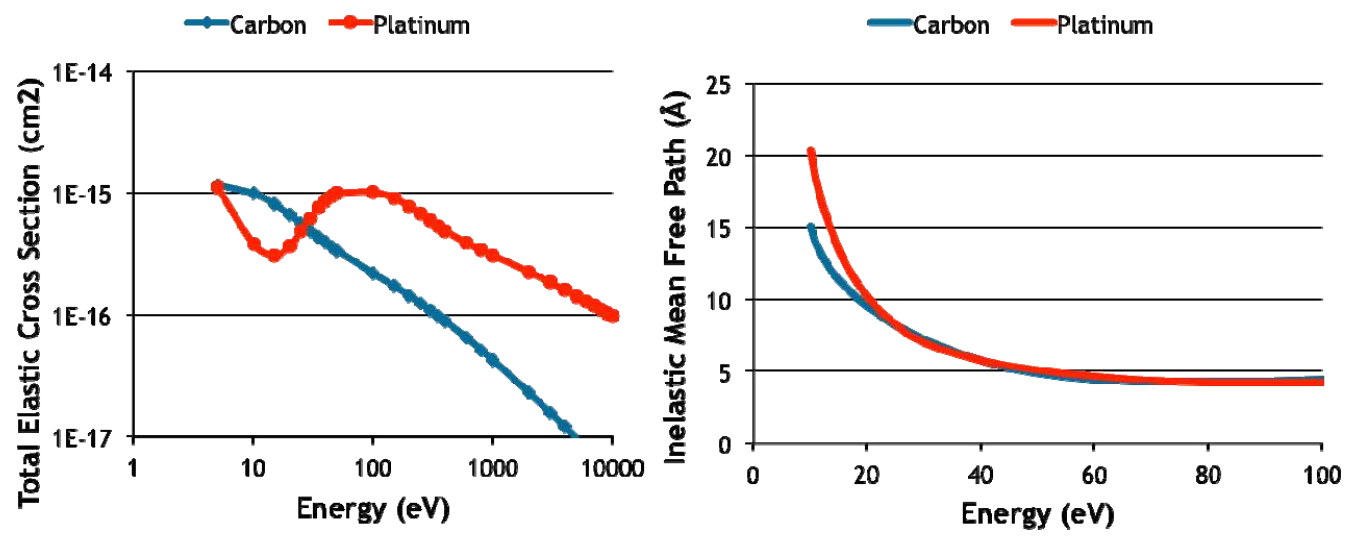

Figure 10 Elastic and inelastic electron scattering for carbon and platinum (data from http://www.ioffe.ru) 
The effect of the metal loading level on the sensitivity of the resist and its patterning performance was investigated for the first metal additive chosen (figure 11). Increasing the level, which is expressed as percentage of the total weight of the resist, from 0 to 4 percent shows a near-linear increase in sensitivity even at these comparatively low levels of addition. Also seen is that the patterning performance slowly decreases as more metal is added. The increase in sensitivity could be a cause of this as we navigate the RLS triangle. Another possibility is that the metal additive interferes with the chemistry of the photoresist and this is under investigation at the moment. The direct measurement of the absorption has also been attempted. For xMT without metal additive the absorption coefficient was measured as 4.3 $\mu \mathrm{m}^{-1}$ and rose to $8.3 \mu \mathrm{m}^{-1}$ with 2 percent metal loading. Experimental details and in depth discussion is reported in [9].

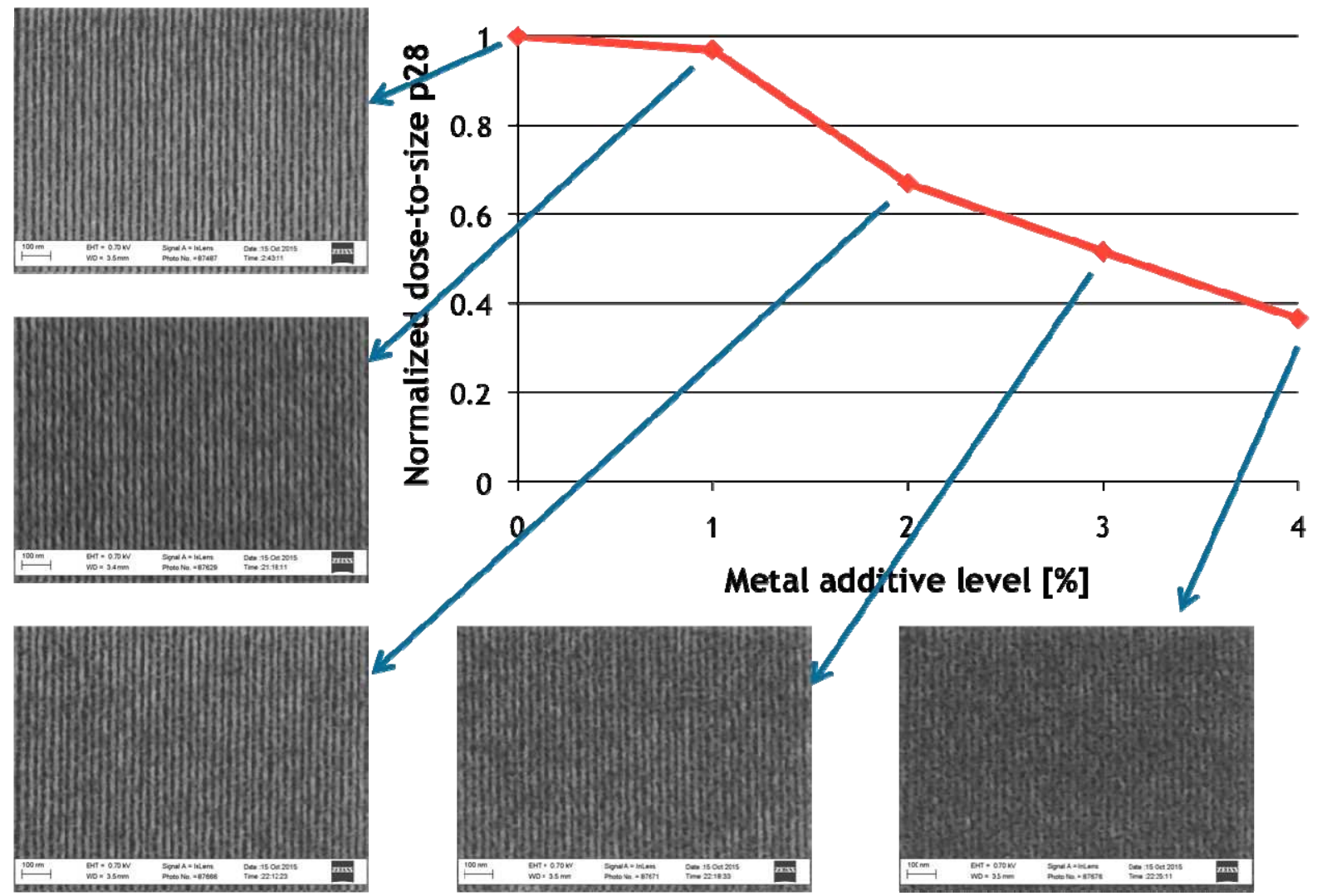

Figure 11 Effect of metal loading level on sensitivity and patterning

So far we have tested about 10 different metal additives and seen a variety of different behavior with the metal addition both increasing and quenching the sensitivity (figure 12). Each symbol represents a different metal. As metals differ drastically in their weight, the data is plotted in numbers of metal atoms rather then weight percentage of addition to be more comparable between them.

\section{CONCLUSION}

We have undertaken a series of studies to optimize the performance of xMT, a new molecular photoresist. A new quencher was identified that allowed to improve the LWR at $14 \mathrm{~nm} \mathrm{hp}$ by fifty percent to $3.56 \mathrm{~nm}$. A cut-off value was identified in the quencher loading level beyond which no further improvement in pattern quality was seen. This allowed the sensitivity to be improved by twenty five percent compared to the baseline resist. The role of xMT in the patterning process was further investigated and showed that the linkage of DBU and xMT functional groups is an important factor. First exposure results on a NXE 3300 scanner were presented with patterns down to $19 \mathrm{~nm}$ hp delineated. With more process improvements it should be possible to improve the resolution further. The concept of metal additives was introduced and sensitivity enhancement demonstrated on an example. Dose improvement of up to sixty percent was seen for relatively low metal levels. We have begun screening different metals for their effect on sensitivity and patterning performance in EUV. 


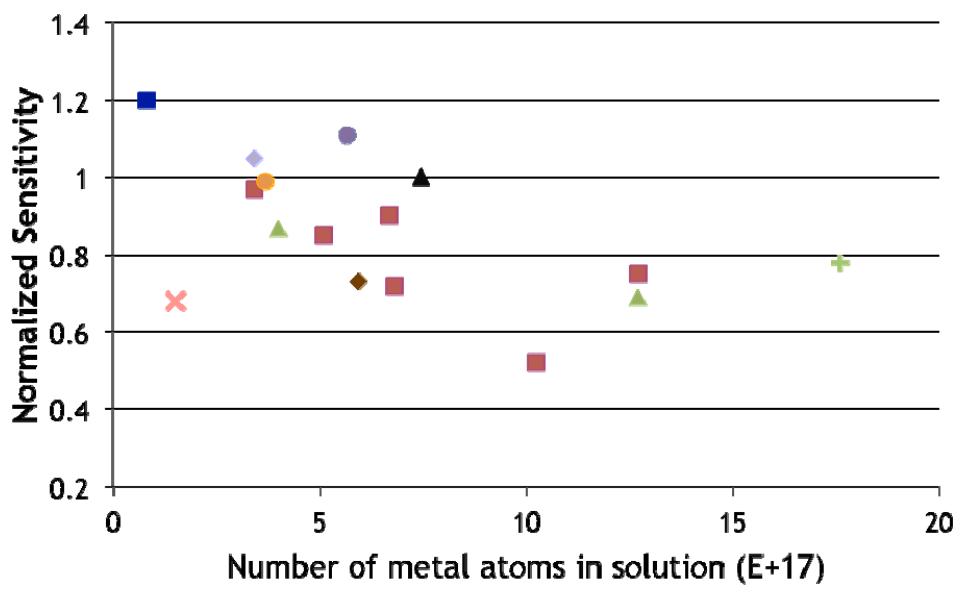

Figure 12 Screening of metals for sensitivity modification

\section{ACKNOWLEDGEMENTS}

The authors would like to thank Ms Michaela Vockenhuber and Mr Daniel Fan from PSI for their assistance with the EUV exposures. Part of this work was performed at Swiss Light Source (SLS), Paul Scherrer Institute, 5232 Villigen, Switzerland. They would further like to thank Danilo de Simone from IMEC for the exposure in the NXE330 and imaging the resulting patterns.

\section{REFERENCES}

[1] Stowers, J. K., Telecky, A., Kocsis, M., Clark, B. L., Keszler, D. A., Grenville, A., Anderson, C. N., Naulleau, P. P., "Directly patterned inorganic hardmask for EUV lithography," Proc. SPIE 7969, 796915 (2011).

[2] Krysak, M., Trikeriotis, M., Schwartz, E., Lafferty, N., Xie, P., Smith, B., Zimmerman, P., Montgomery, W., Giannelis, E., Ober, C. K., 'Development of an inorganic nanoparticle photoresist for EUV, e-beam, and $193 \mathrm{~nm}$ lithography," Proc. SPIE 7972, 79721C (2011).

[3] Cardineau, B., Del Re, R., Al-Mashat, H., Marnell, M., Vockenhuber, M., Ekinci, Y., Sarma, C., Neisser, M., Freedman, D. A., Brainard, R. L., "EUV resists based on tin-oxo clusters," Proc. SPIE 9051, 90511B (2014).

[4] Päivänranta B., Langner A., Kirk E., David C., Ekinci Y., "Sub-10 nm patterning using EUV interference lithography," Nanotechnology Vol. 22, 375302 (2011).

[5] Ekinci Y., Vockenhuber M., Terhalle B., Hojeij M., Wang L., Younkin T. R., "Evaluation of resist performance with EUV interference lithography for sub-22 nm patterning," Proc. SPIE 8322, 83220W (2012).

[6] Frommhold, A., McClelland, A., Yang, D. X., Palmer, R. E., Roth, J., Ekinci, Y., Rosamund, M. C., Robinson, A. P. G., "Towards $11 \mathrm{~nm}$ half-pitch resolution for a negative-tone chemically amplified molecular resist platform for extreme-ultraviolet lithography," Proc. SPIE 9425, 942504 (2015).

[7] Lawson, R. A., Chun J. S., Neisser, M. et al., "Methods of controlling cross-linking in negative-tone resists," Proc. SPIE 9051, 9051Q (2014).

[8] Frommhold, A., Yang, D. X., McClelland, A., Xue, X., Ekinci, Y., Palmer, R. E., Robinson, A. P. G., "Optimization of Fullerene-based Negative tone Chemically Amplified Fullerene Resist for Extreme Ultraviolet Lithography," Proc. SPIE 9051905119 (2014).

[9] Fallica, R. A., Stowers, J. K., Grenville, A., Frommhold, A., Robinson, A. P. G., Ekinci, Y., "Dynamic absorption coefficients of CAR and non-CAR resists at EUV," Proc. SPIE 9776 (2016) (submitted). 